\title{
Toward Fast and Reliable Potential Energy Surfaces for Metallic Pt clusters by Hierarchical Delta Neural Networks (supporting information)
}

\author{
Geng Sun ${ }^{1}$, Philippe Sautet*1,2
}

1. Department of Chemical and Biomolecular Engineering, University of California, Los Angeles, Los Angeles, California 90095, United States

2. Department of Chemistry and Biochemistry, University of California, Los Angeles, Los Angeles, California 90095, United States

\section{Symmetrically increase the number of descriptors.}

Two different kinds of descriptors are used throughout this paper. The first one is

\subsection{Chebyshev polynomials}

The Chebyshev polynomials are a set of orthogonal polynomials defined in the range of $[0,1]$. The detailed description of exploiting Chebyshev polynomials to extract chemical environment is proposed by Artrith et al. ${ }^{1}$ The essential idea is that the radial distribution function $R D F_{i}$ and angular distribution function $A D F_{i}$ (of atom $i$ ) can be expanded by transformed Chebyshev polynomials.

$$
R D F_{i}=\sum_{m} c_{m}^{r} f_{m}(r)
$$

As well as

$$
A D F_{i}=\sum_{m} c_{m}^{a} f_{m}(r)
$$


The expansion coefficients $c_{m}^{r}$ and $c_{m}^{a}$ can be used to reconstruct the RDF and ADF, hence it is also able to reconstruct the geometry of quantum system. In the end, the number of coefficients are totally determined by the highest order of Chebyshev polynomials used for the expansion. We investigated different combinations and number of total input layers (shown in Table $\mathrm{S}$ ).

\subsection{Symmetry function}

There are two types of symmetry functions. The first one is the radial part $G_{i}^{2}$ :

$$
G_{i}^{2}=\sum_{j=1}^{N} e^{-\eta\left(r_{i j}-R_{S}\right)^{2}} f_{c}\left(r_{i j}\right)
$$

Where the $r_{i j}$ is the distance between atom $i$ and its neighbor $j . f_{c}\left(r_{i j}\right)$ is a cutoff function, which vanishes at the chosen cutoff distance $R_{C}\left(R_{C}=6.5 \AA\right.$ in this paper). $R_{S}$ is a parameter to shift the center of the Gaussian-like function from the position of atom $i$ to its nearby. The second group of symmetry functions is describing the angular distribution of bonds angles $G_{i}^{4}$ :

$$
G_{i}^{4}=2^{1-\zeta} \sum_{j, k}\left(1+\lambda \cos \theta_{i j k}\right)^{\zeta} e^{-\eta\left(r_{i j}^{2}+r_{j k}^{2}+r_{k i}^{2}\right)} f_{c}\left(r_{i j}\right) f_{c}\left(r_{k j}\right) f_{c}\left(r_{i k}\right)
$$

In equation $S 4, \zeta, \lambda$ and $\eta$ are parameters.

We followed the procedures proposed by Imbalzano et al. ${ }^{2}$ to select $\eta$. The procedures are briefly recalled below. Firstly, we choose cutoff as $R_{c}$ and choose the number of intervals $(n)$ to divide the $R_{c}$ equally. We can obtain $\mathrm{m}=(0,1,2,3, . . \mathrm{n})$ and the corresponding $\eta_{m}$ is:

$$
\eta_{m}=\left(\frac{n^{\frac{m}{n}}}{R_{c}}\right)^{2}
$$

One can obtain $\mathrm{n}+1 \eta$ values from equation $\mathrm{S} 5$.

For each $\mathrm{m}=(1 \ldots \mathrm{n}-1)$, one also generate a pair of $\eta$ and $R_{s}$ for $G_{i}^{2}$ by: 


$$
\begin{gathered}
R_{s}^{m}=\frac{R_{c}}{n^{\frac{m}{n}}} \\
\eta_{s}^{m}=\frac{1}{\left(R_{s}^{n-m}-R_{s}^{n-m-1}\right)^{2}}
\end{gathered}
$$

Therefore, we can obtain another n-2 values for $G_{i}^{2}$.

For $G_{i}^{4}$ descriptors, one can use the same procedures shown in Equation $S 5$ and fixed $\lambda=[+1,-1]$ and $\zeta=[1,4,16]$. In the end, one can obtain symmetry function values by just chose the number of intervals $\mathrm{n}$ for radial part and angular part individually.

Table S1. The number of intervals used in radial and angular part of symmetry functions and the total size of the symmetry function descriptors. For some choices of nradial and nangular, they will result in the same size of descriptors. Only the best combination is shown in Figure 1 and Figure 4 in the manuscript. RMSE indicates the error at the $5000^{\text {th }}$ iteration for the trainset and its unit is meV/atom. The cutoff for symmetry function descriptors are always 6.5 Angstrom. From the number of the nradial and nangular (as well as the cutoff) the $\eta$ for symmetry function is uniquely defined through equation $\mathrm{S} 1$ to equation $\mathrm{S} 7$.

\begin{tabular}{|c|c|c|c|c|c|c|c|c|c|}
\hline size & nrad & nang & RMSE & nrad & nang & RMSE & nrad & nang & RMSE \\
\hline 22 & 2 & 2 & 17.735 & & & & & & \\
\hline 24 & 3 & 2 & 17.168 & & & & & & \\
\hline 26 & 4 & 2 & 16.246 & & & & & & \\
\hline 28 & 5 & 2 & 14.587 & 2 & 3 & 15.228 & & & \\
\hline 30 & 6 & 2 & 13.997 & 3 & 3 & 14.607 & & & \\
\hline 32 & 7 & 2 & 13.793 & 4 & 3 & 13.957 & & & \\
\hline 34 & 5 & 3 & 13.561 & 8 & 2 & 13.859 & 2 & 4 & 13.88 \\
\hline 36 & 6 & 3 & 12.887 & 3 & 4 & 13.691 & & & \\
\hline 38 & 7 & 3 & 12.522 & 4 & 4 & 13.136 & & & \\
\hline
\end{tabular}




\begin{tabular}{|c|c|c|c|c|c|c|c|c|c|}
\hline 40 & 5 & 4 & 12.69 & 8 & 3 & 12.731 & 2 & 5 & 13.361 \\
\hline 42 & 6 & 4 & 12.259 & 3 & 5 & 13.081 & & & \\
\hline 44 & 7 & 4 & 11.811 & 4 & 5 & 12.605 & & & \\
\hline 46 & 8 & 4 & 12.086 & 5 & 5 & 12.248 & 2 & 6 & 13.202 \\
\hline 48 & 6 & 5 & 11.64 & 3 & 6 & 13.004 & & & \\
\hline 50 & 7 & 5 & 11.567 & 4 & 6 & 12.446 & & & \\
\hline 52 & 8 & 5 & 11.333 & 5 & 6 & 12.338 & 2 & 7 & 13.34 \\
\hline 54 & 6 & 6 & 11.803 & 3 & 7 & 13.082 & & & \\
\hline 56 & 7 & 6 & 11.54 & 4 & 7 & 12.622 & & & \\
\hline 58 & 8 & 6 & 11.794 & 5 & 7 & 12.284 & 2 & 8 & 13.13 \\
\hline 60 & 6 & 7 & 11.922 & 3 & 8 & 13.185 & & & \\
\hline 62 & 7 & 7 & 11.506 & 4 & 8 & 12.381 & & & \\
\hline 64 & 8 & 7 & 11.556 & 5 & 8 & 12.151 & & & \\
\hline 66 & 6 & 8 & 11.982 & & & & & & \\
\hline 68 & 7 & 8 & 11.503 & & & & & & \\
\hline 70 & 8 & 8 & 11.377 & & & & & & \\
\hline
\end{tabular}

Table S 2 The radial and angular orders of Chebyshev polynomials. Size of $X$ is also the size of input layers excluding the bias node.

\begin{tabular}{|l|l|l|l|l|l|}
\hline \multicolumn{7}{|l|}{ Chebyshev polynomials (Cutoff 8.0 Angstrom) } \\
\hline N(Radial) & 16 & 24 & 32 & 40 & 48 \\
\hline N(Angular) & 8 & 16 & 24 & 32 & 40 \\
\hline Size of X & 26 & 42 & 58 & 74 & 92 \\
\hline
\end{tabular}




\begin{tabular}{|l|l|l|l|l|l|}
\hline N(Radial) & 16 & 24 & 32 & 40 & 48 \\
\hline N(Angular) & 16 & 24 & 32 & 40 & 48 \\
\hline Size of X & 34 & 50 & 66 & 82 & 96 \\
\hline
\end{tabular}

\section{Early stop scheme to select the training results}

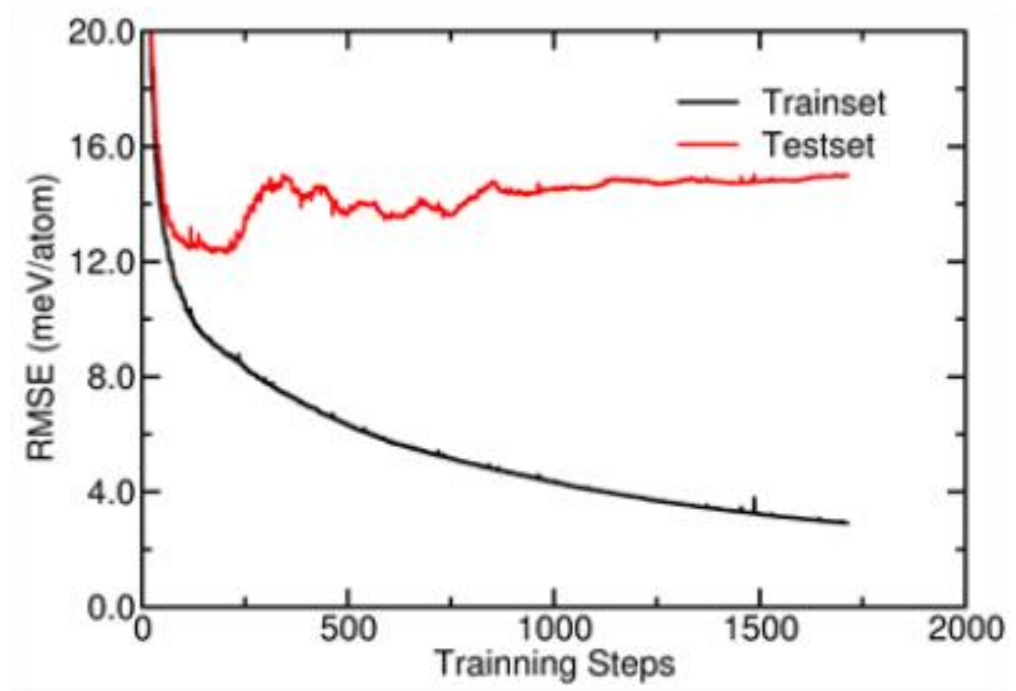

(a) demonstration of early-stop scheme

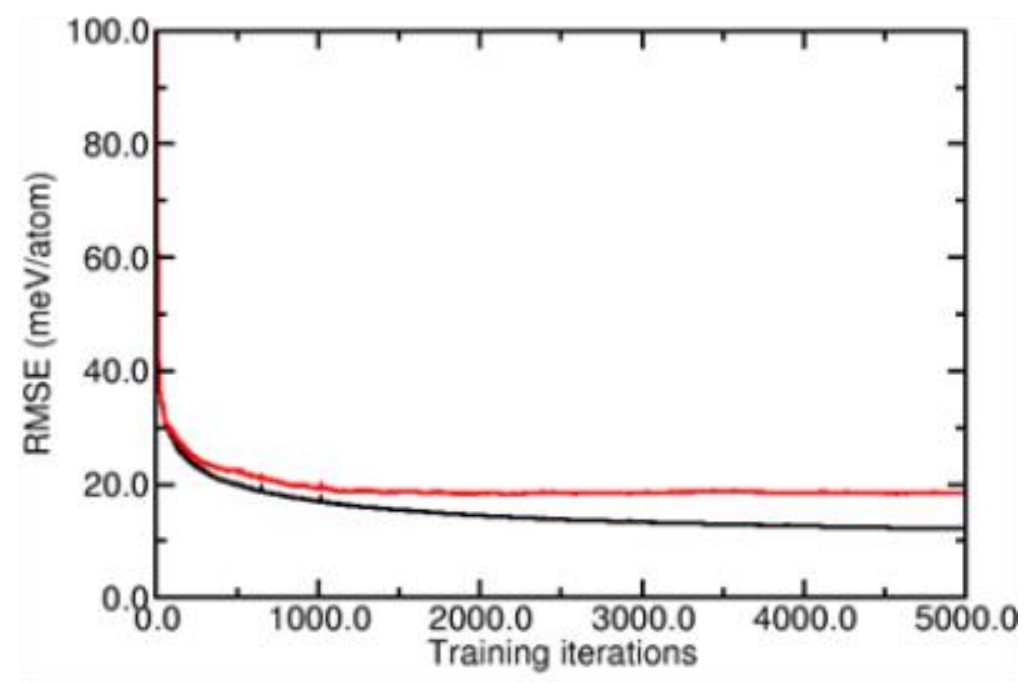

(b) an example of RMSE evolution with increasing training steps

Figure S 1 (a) An example to demonstrate the early-stop scheme to select the training results. Initially, both of the training errors and testing errors decrease rapidly, while the errors of the testing stet achieve a minimum at around 200 steps and constantly arise after that step. Therefore, the optimal results for each training use the step who shows minimal testing errors. That corresponds to the $200^{\text {th }}$ step in this figure. (b) 
Evolution of the RMSE with number of training steps when using symmetry functions and nrad=8,nang=4. The black line indicates RMSE of the trainset and the red line indicates the RMSE of the testset (control set). One can see that the RMSE is almost constant after 3000 step. (the early stop scheme selects the 2182th iteration).

\section{Comparison between DFT with single zeta basis sets and references}
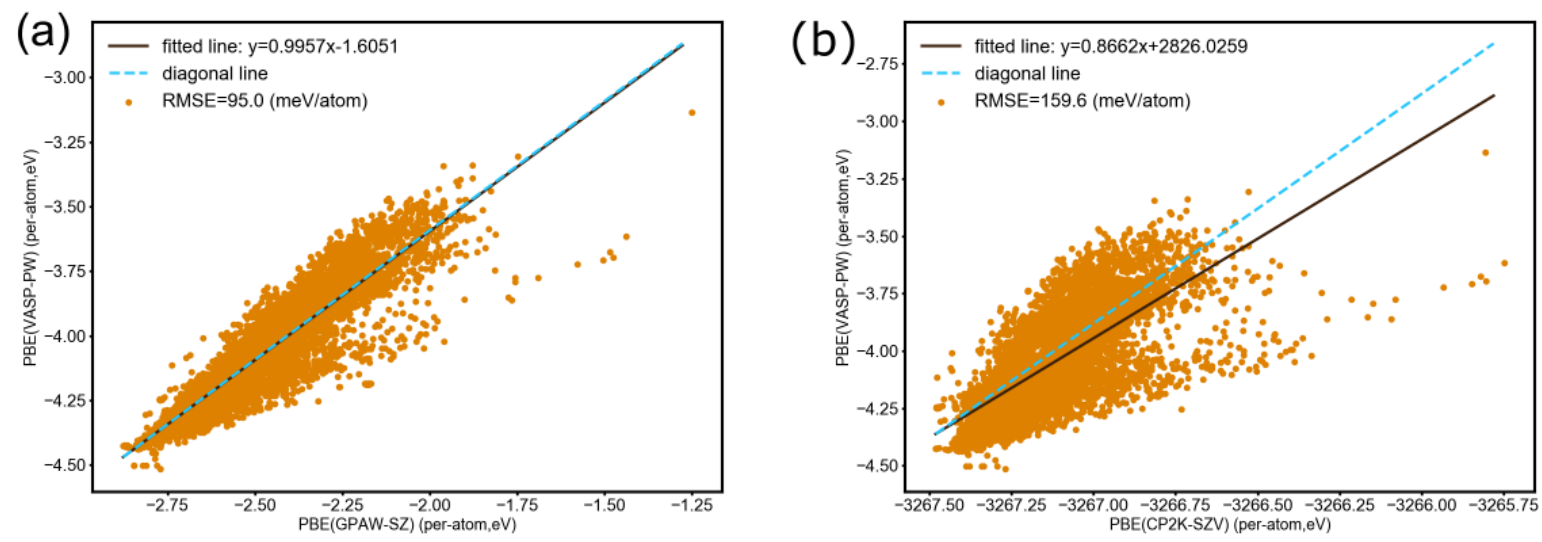

Figure $S 2$ The comparison between the reference database PBE(VASP-PW) and two different low level DFT methods. PBE(GPAW-SZ) (a) and PBE(CP2K-SZ) (b). Blue dash lines indicate the diagonal lines. Black lines indicate the fitted equations from energies obtained by the two methods. The RMSE shown in the figures are computed by $R M S E=\sqrt{\frac{1}{N} \sum\left(\Delta E_{i} / n_{i}\right)^{2}} \cdot \Delta E_{i}=a E_{i}^{S Z}+b-E_{i}^{r e f}$. a and $\mathrm{b}$ are the fitted coefficients shown in the figures. 


\section{Comparison between energies from different functional.}

(a)

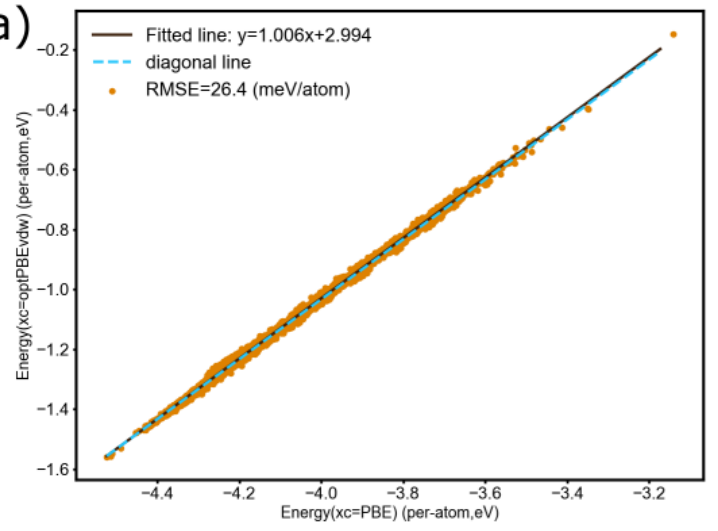

(b)

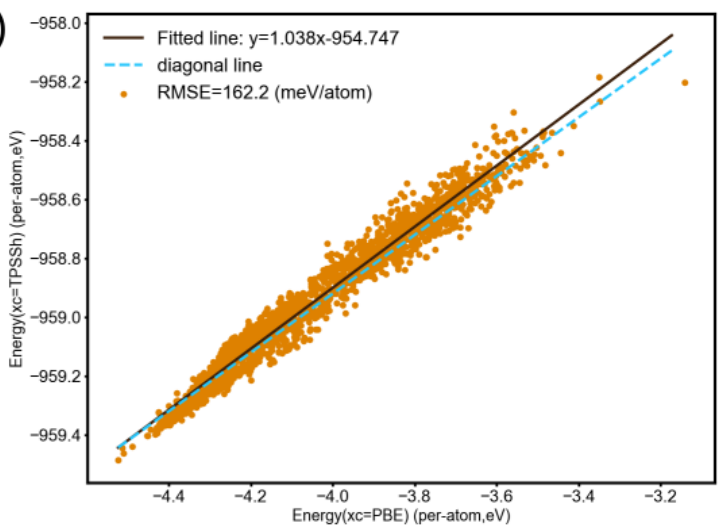

Figure S 3 Comparison of energies computed from different functionals. Subfigure (a) compares the difference between the optPBE-vdW functional and pure PBE. Subfigure (b) compares the TPSSh functional with PBE. All the calculations are conducted with VASP package with plane waves up to 250 $\mathrm{eV}$. Non spin polarized calculations are used. 


\section{Comparison of using PBE(CP2K-SZV) and GPAW(SZ) as low level DFT calculation.}

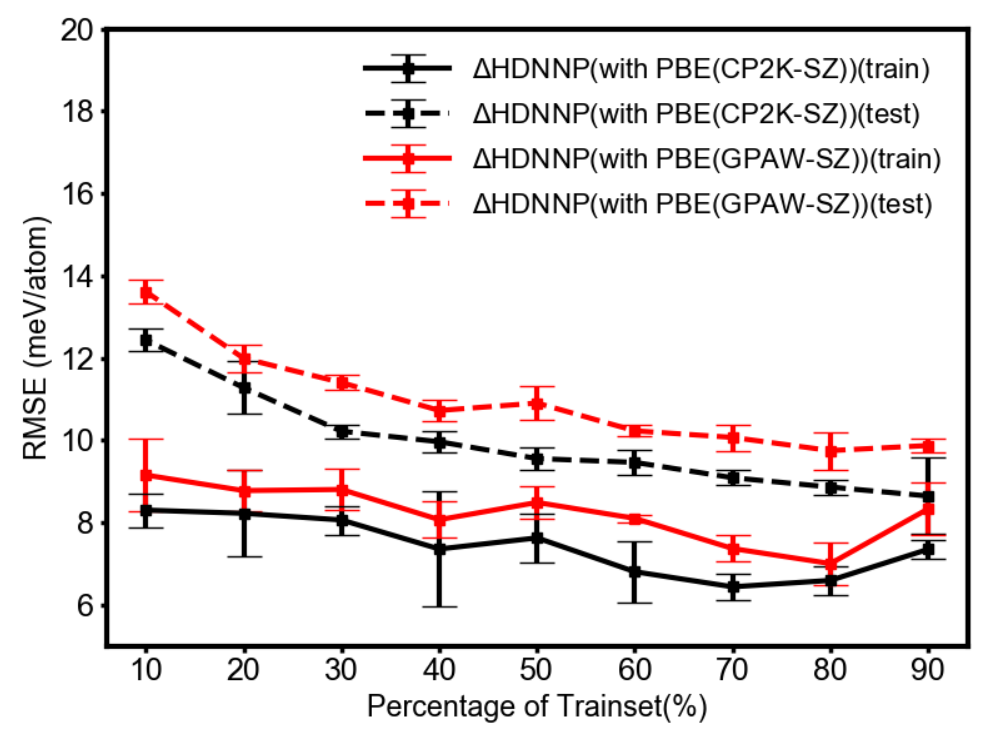

Figure S 4 Compare performance of $\triangle \mathrm{HDNNP}$ with $\mathrm{PBE}(\mathrm{CP} 2 \mathrm{~K}-\mathrm{SZ})$ as low-accuracy DFT and with $\mathrm{PBE}(\mathrm{GPAW}-\mathrm{SZ}$ ) as low-accuracy DFT. In this test, the reference DFT calculation is PBE(VASP-PW).

\section{TPSS functional as high-level target.}

(a)

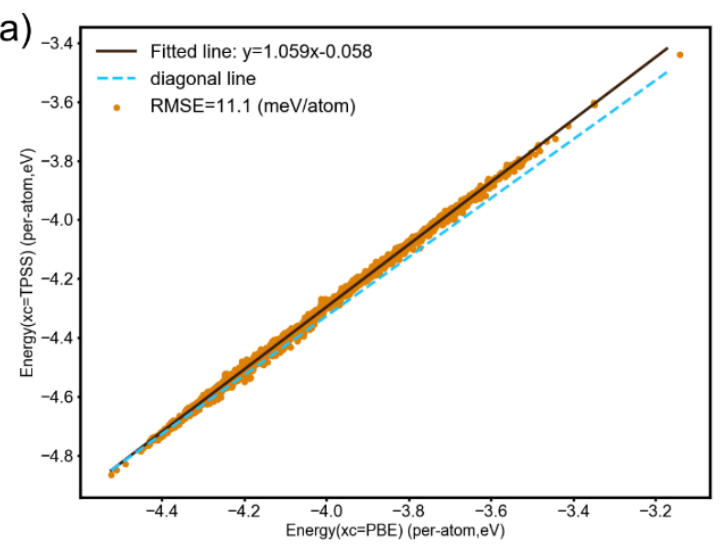

(b)

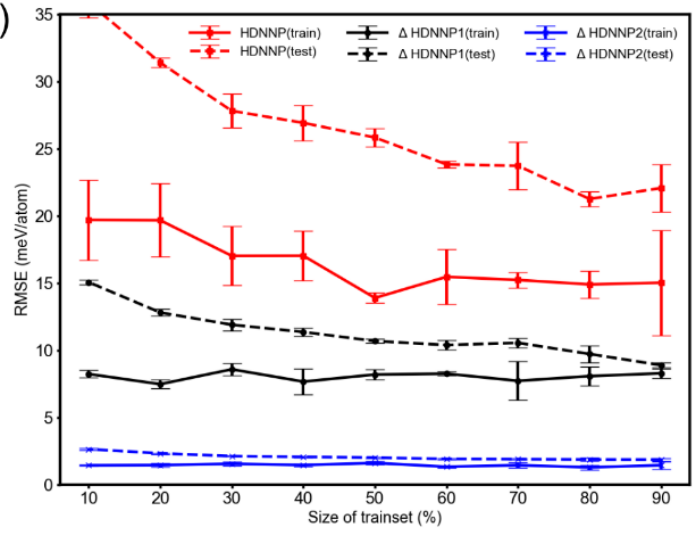

Figure S 5 Using $\triangle$ HDNNP to train difference between PBE and TPSS. (a) comparison between the energies of TPSS and PBE (b) $\triangle$ HDNNP and HDNNP training results. The reference method is TPSS(VASP-PW). Red lines are HDNNP results without low-level DFT. $\triangle$ HDNNP1 (black lines) shows 
the results of $\triangle \mathrm{HDNNP}$ using $\mathrm{PBE}(\mathrm{CP} 2 \mathrm{~K}-\mathrm{SZ})$ as low level method. $\triangle \mathrm{HDNNP} 2$ (blue lines) shows the results of $\triangle$ HDNNP using PBE(VASP-PW) as low level DFT method. $\mathrm{x}$ axis is the percentage of trainset in the database and all the other structures (not used in trainings) are used as testing set. Sold lines are RMSEs of trainsets. Dash lines are RMSEs of testsets.

\section{Quality of force in HDNNP and $\triangle$ HDNNP}
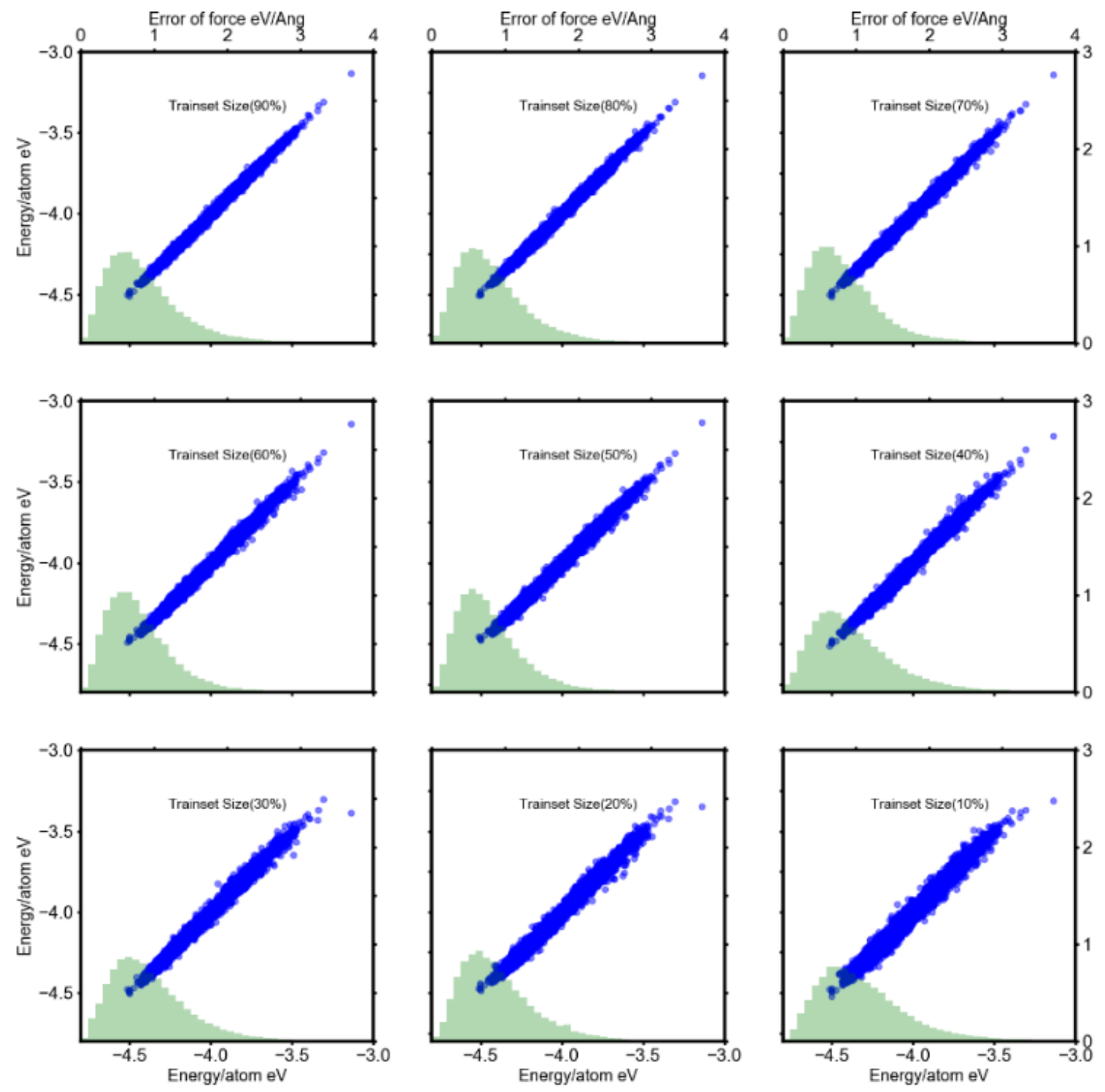

Figure S 6. Comparison of the energies and forces in the reference database and computed by trained HDNNP. The blue scatters show the comparison of averaged atomic energy (left axis and bottom axis). The green bars show the distribution of the magnitude of force difference $\left|\overrightarrow{F_{\text {ref }}}-\overrightarrow{F_{H D N N P}}\right|$ (right and top axis). All the data (including the trainset and testset) is included. Input layer size is 46 computed by symmetry functions. The architecture of the neural network is 46-30-30-1. 

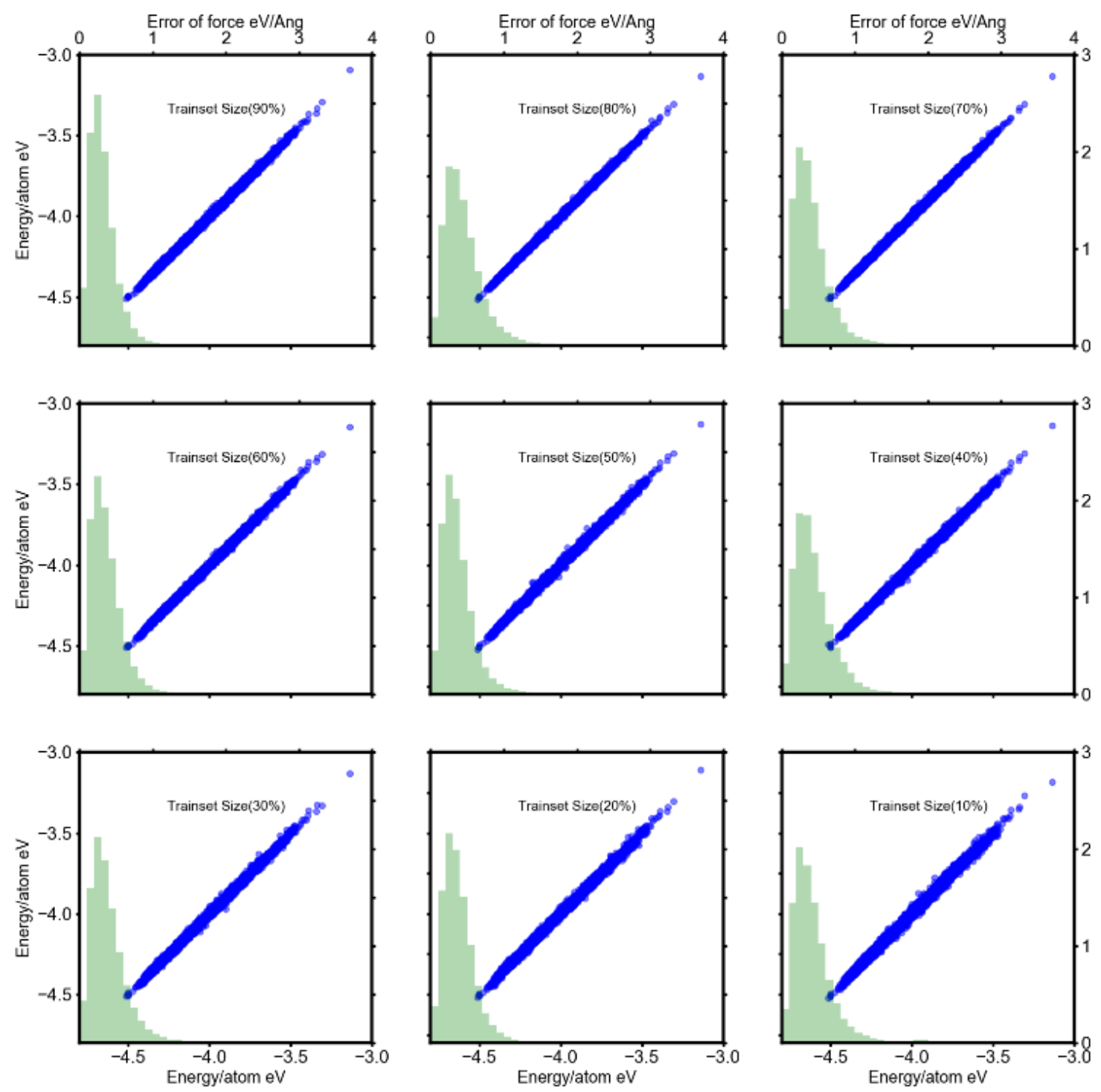

Figure S 7 Comparison of the energies and forces in the reference database and computed by trained $\triangle$ HDNNP. The blue scatters show the comparison of averaged atomic energy (left axis and bottom axis). The green bars show the distribution of the magnitude of force difference $\left|\overrightarrow{F_{r e f}}-\overrightarrow{F_{H D N N P}}\right|$ (right and top axis). All the data (including the trainset and testset) is included. Input layer size is 46 computed by symmetry functions. The architecture of the neural network is 46-30-30-1. 


\section{Basis sets convergence for plane waves and finite difference methods with GPAW package}
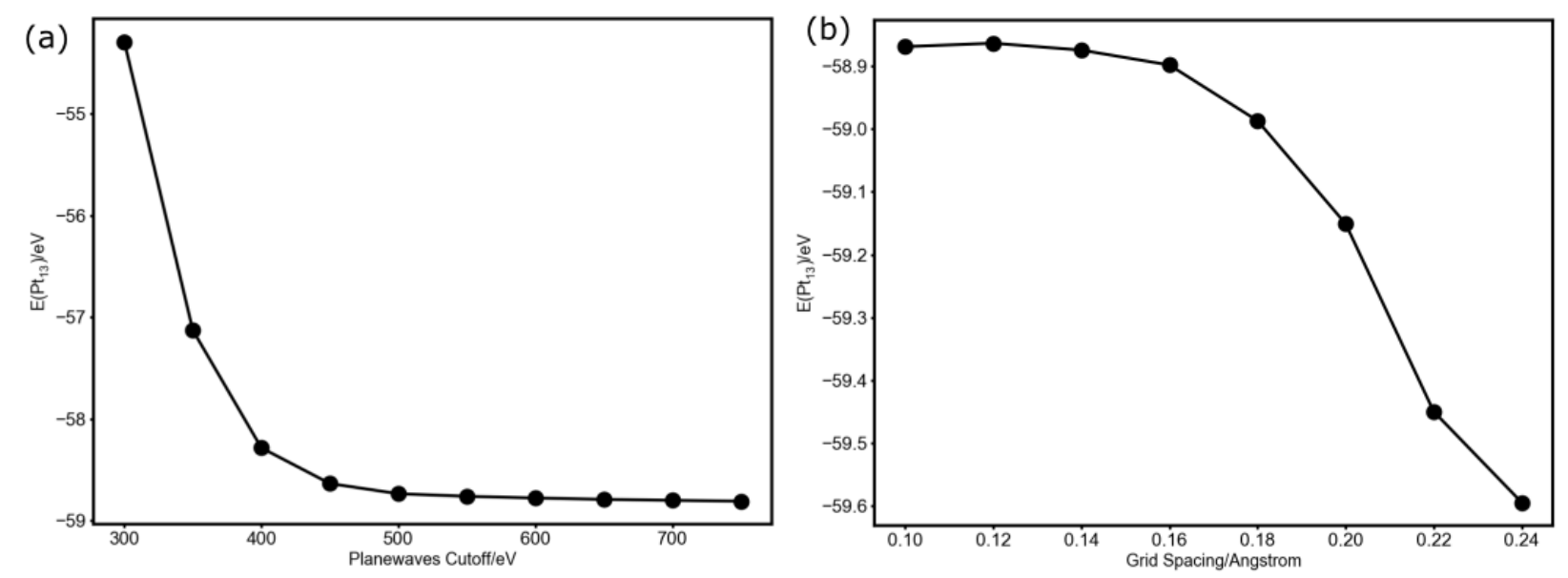

Figure S 8 Converging the plane wave basis sets (in the plane wave mode) and grid spacing (in the finite difference mode) for GPAW DFT calculations. This calculation is conducted with a $\mathrm{Pt}_{13}$ cluster as an example. Finally, we choose cutoff as $450 \mathrm{eV}$ for the GPAW plane wave (PW) mode, which provides a small error around $10 \mathrm{meV} /$ atom compared with higher cutoffs. GPAW also provides another method called finite difference (FD) mode, which also provides a systematic way to increase the calculation accuracy by decreasing the grid spacing h. Therefore, we also used the FD mode to compare CPU efficiency in DFT between a single zeta basis set and fully converged basis sets (PW mode or FD mode). In the calculation, we choose $\mathrm{h}=0.18$ Angstrom in FD mode. 


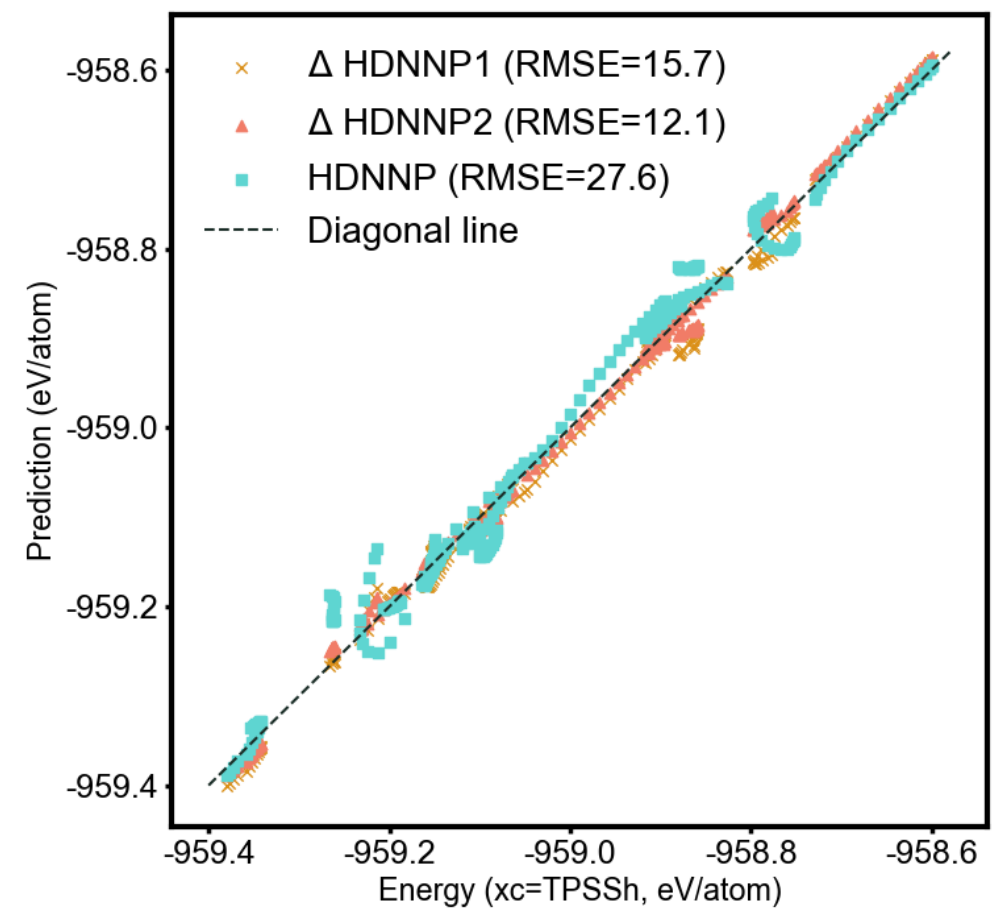

Figure S 9 Benchmark the accuracy of $\triangle \mathrm{HDNNP1}$ (with PBE(CP2K-SZ) as approximate DFT), $\triangle$ HDNNP2 (with PBE(VASP-PW) as approximate DFT) and HDNNP targeting at energies from TPSSh functional. The units of the RMSE is meV/atom.

\section{Averaged atomic energy distribution for different database}

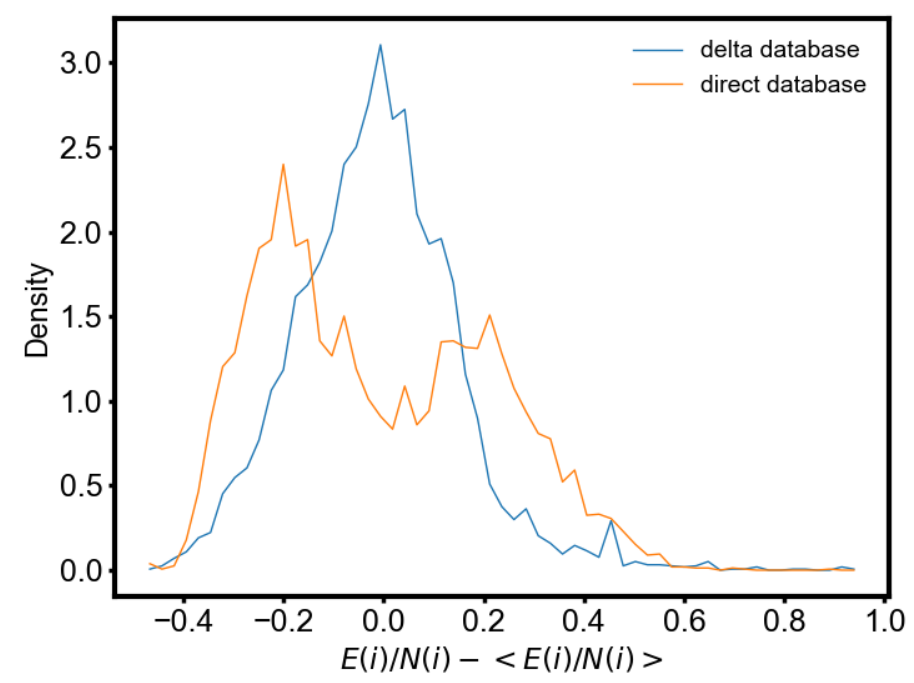

Figure S 10 The distribution of averaged atomic energies. The $x$ axis is $E(i) / N(i)-\langle E(i) / N(i)\rangle$, in which $\mathrm{E}(\mathrm{i})$ is the reference energies from DFT calculations (or delta energies from two different DFT calculations) for structure $\mathrm{i}$ and $\mathrm{N}(\mathrm{i})$ is the number of atoms for structure $\mathrm{i} . \mathrm{y}$ axis is the normalized 
distribution. In this figure the delta database is constructed from the PBE(GPAW-SZ) and PBE(VASPPW) and the direct database refers to the PBE(VASP-PW).

\section{Violin plot of the fingerprints distribution in the database.}
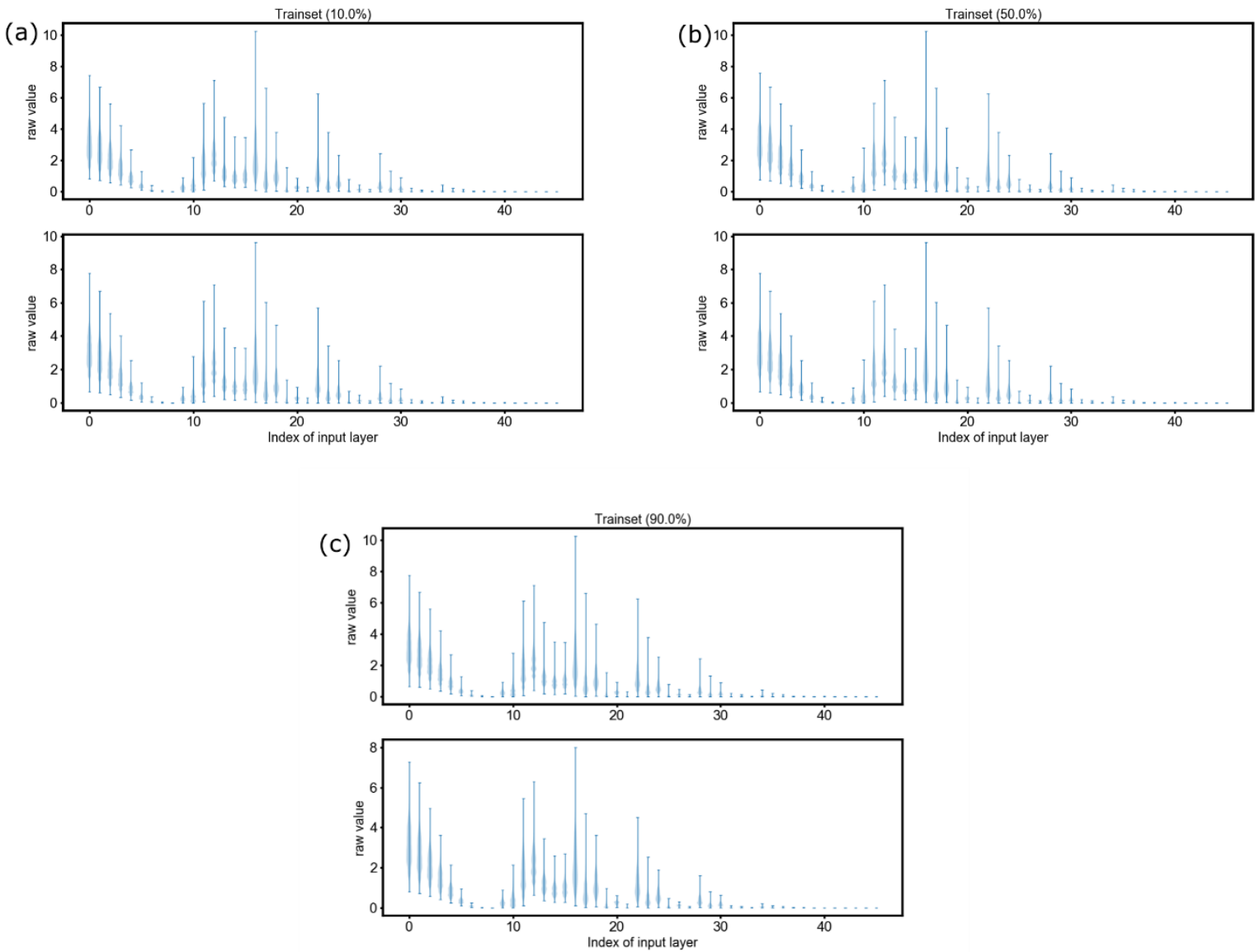

Figure S 11 The violin plot of the distribution of the feature vectors (using symmetry functions). The $\mathrm{x}$ axis is the index and $y$ is the value of the descriptors. The shade is the distribution of the $y$ values. For each case, the trainset is randomly selected using $10 \%, 50 \%$ and $90 \%$ of the whole database respectively.

\section{PCA analysis with input layer or output of last hidden layer from trained neural network potentials}



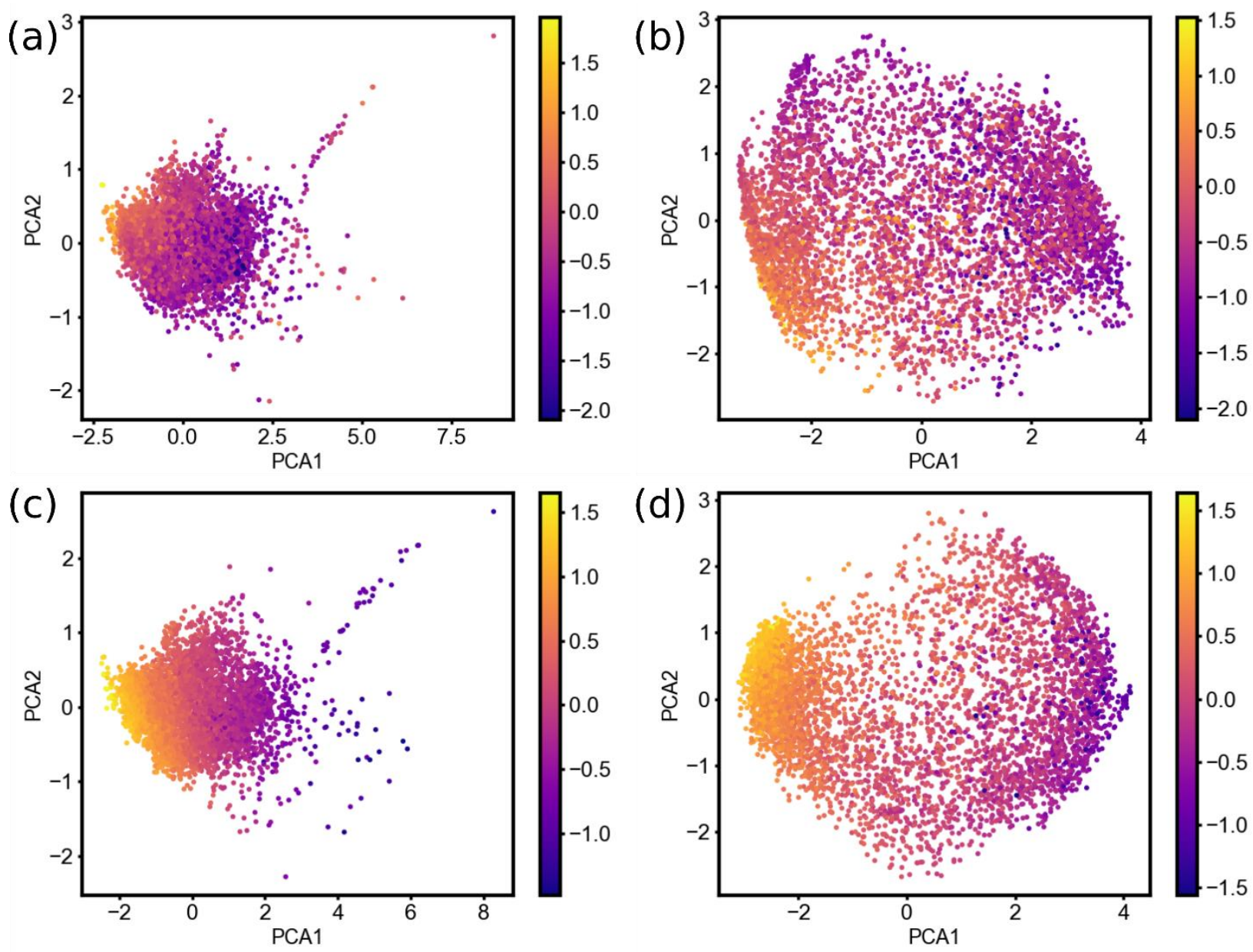

Figure S 12 PCA analysis (a), (c) with symmetry function values as inputs or (b), (d) with the output of last hidden layer of neural network as inputs. In all the case, neural network learned atomic energies are used as color code; (a), (b) using HDNNP learned atomic energies as color code, (c), (d) using $\triangle$ HDNNP learned atomic energies as color code. Both of them use $90 \%$ of the database as trainset and the architecture of the neural network potential is 46-(2x30)-1. Only $7 \%$ of the atoms (randomly selected) are shown in the figures for clarity purposes. 


\section{Ranges of feature vectors in out-of-sample testset.}
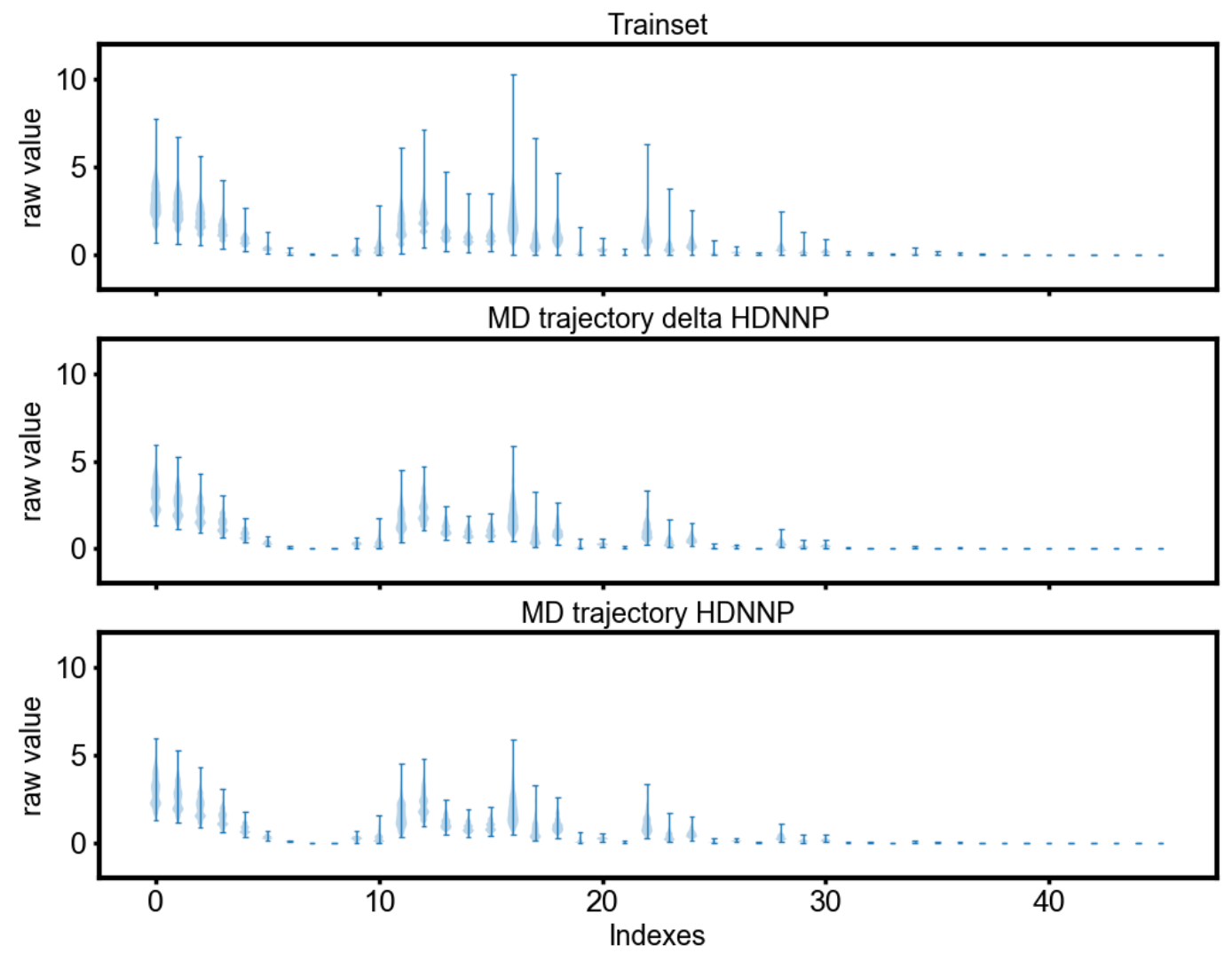

Figure S 13 The violin plot for the distribution of the feature vectors. The $\mathrm{x}$ axis is the index and $\mathrm{y}$ is the value of the descriptors. The shade is the distribution of the y values. The top graph represents the feature vectors of the training database. The middle graph shows the feature vectors from MD trajectories, which are generated by delta HDNNP. The bottom graph shows feature vectors from MD trajectories generated by the direct HDNNP.

\section{References}

1. Artrith, N.; Urban, A.; Ceder, G., Efficient and Accurate Machine-Learning Interpolation of Atomic Energies in Compositions with Many Species. Phy. Rev. B 2017.

2. Imbalzano, G.; Anelli, A.; Giofre, D.; Klees, S.; Behler, J.; Ceriotti, M., Automatic Selection of Atomic Fingerprints and Reference Configurations for Machine-Learning Potentials. J. Chem. Phys. 2018, $148,241730$. 